\title{
HEALTH AND DISEASE PROFILE OF CHILDREN IN REMOTE RURAL POCKETS OF HIMACHAL PRADESH, WITH A LOOK-OUT FOR UNCOMMON AND RARE DISEASES- A COLLAGE OF HEALTH CAMP BASED CROSS SECTIONAL STUDY
}

\author{
Rita Hajela ${ }^{1}$
}

${ }^{1}$ Associate Professor, Department of Paediatrics, Maharishi Markandeshwar Medical College and Hospital, Kumarhatti, Solan, Himachal Pradesh, India.

\begin{tabular}{l}
\hline ABSTRACT \\
BACKGROUND \\
Health camp organization is an established mode of health care delivery to unreached rural population deprived of specialist care. \\
Himachal Pradesh is a hilly area with difficult to travel geographical terrain. Our institute organized multispecialty health camps in \\
remote rural pockets of Himachal Pradesh including paediatrician's services.
\end{tabular}

\section{METHODS}

Health status and disease profile of children who attended these camps were entered in a predesigned proforma. Haemoglobin estimation was done on the spot. Data of 472 children attending 20 such camps were analysed.

\section{RESULTS}

$7.84 \%$ children came for paediatric consultation from 6019 patients who attended these camps. Maximum number of patients were in age group of 6-12 years. $25.6 \%$ children had a weight for age $<3^{\text {rd }}$ percentile and an almost a similar number of $25.4 \%$ had height less than $<3$ rd percentile for age, suggestive of malnutrition and short stature respectively. $78.8 \%$ children had anaemia of varying degree. $36.7 \%$ children were brought with long duration of illness lasting for more than one year and another $5.51 \%$ had problems since birth. The most common presenting complaint was pain abdomen with gastrointestinal tract, the most commonly involved system. Children suffering with uncommon \& rare diseases, various Syndromes and CNS diseases were also found hidden in these rural pockets without any diagnostic or therapeutic help.

\section{CONCLUSIONS}

The study highlights a delayed health care seeking behaviour in rural children and parents. Anaemia is still a big problem in spite of many health programs in place for decades. Protein energy malnutrition is prevalent, and it is manifesting in the form of stunting in almost $25 \%$ of attended child population. A paediatrician-oriented specialist care for early diagnosis and management of these children is the need of hour. A lookout for uncommon diseases is required to address this issue in India to keep pace with global health care approach.

\section{KEY WORDS}

Rural Population, Anaemia, Malnutrition, Health Status, Growth and Development, Rare Diseases, Child, Health Promotion, Genetic Disease

HOW TO CITE THIS ARTICLE: Hajela R. Health and disease profile of children in remote rural pockets of Himachal Pradesh, with a look-out for uncommon and rare diseases- a collage of health camp based cross sectional study. J. Evolution Med. Dent. Sci. 2019;8(24):1914-1920, DOI: 10.14260/jemds/2019/421

\section{BACKGROUND}

Multi-specialty health camp organization by governmental and non-governmental organizations is an established mode of health care delivery to underprivileged unreached rural population.[1,2,3,4]

A lot has been done for upliftment of general health of children, with efforts directed towards common childhood diseases and communicable diseases. Preventive steps have been taken with improved immunization services, special compaigns and including more preventable diseases in National Immunization Schedule.

'Financial or Other Competing Interest': None.

Submission 15-04-2019, Peer Review 03-06-2019,

Acceptance 10-06-2019, Published 17-06-2019.

Corresponding Author:

Dr. Rita Hajela,

Associate Professor,

Department of Paediatrics,

Maharishi Markandeshwar Medical College and Hospital,

Kumarhatti, Solan-173229, Himachal Pradesh, India.

E-mail: hajelarita@gmail.com

DOI: $10.14260 /$ jemds $/ 2019 / 421$

\section{(c) (1) $\left(\frac{\mathrm{BV}}{\mathrm{BV} \mathrm{ND}}\right.$}

Different National and international programs are in place targeting different diseases for quite some time.[5,6] With reduction in childhood communicable diseases, eradication of Polio, control of diarrheal diseases, better survival of premature, reduced neonatal, perinatal and infant mortality, improved nutritional status through mid-day meals, anaemia prophylaxis etc., the spectrum of diseases and overall health status is expected to change. We need to know the present situation for future planning. This study was undertaken to find out the current scenario of health and disease profile of children in unreached remote rural pockets of Himachal Pradesh.

Himachal Pradesh is a hilly area with difficult geographical terrain. Transportation is tough and inconvenient in rural areas and reaching for specialist's care is burdensome, demanding a lot of investment in time, money, physical endurance and mental patience. Chronic illnesses, CNS disorders, Genetic defects and rare diseases are accepted with empathy \& sympathy without much indulgence in modern medicine, optimization of care and possible changes in outcome. Not much data is available regarding such diseases in India, especially from Himachal Pradesh and 
its rural pockets. Whatever data is available, it is through hospital-based studies.[7,8] Our institution MMUC\&H is committed to conducting specialist targeted health camps since its inception, in remote rural areas of Himachal Pradesh, with thin scarce scattered unreached population,

We have tried to pay attention to lesser addressed issues of uncommon findings, dysmorphic features, rare diseases ${ }^{[9,10]}$ or chronic neurological problems.[11,12] marked with limited therapy, poor outcome, desperate situation, superimposed with high economic, physical and mental burden to family, along with its detrimental effect on micro economy of the country. We took into consideration various parameters of health in detail in children attending such health camps, to find out current status of health and disease profile of children in these remote areas as a measure of existing national health policy and for future reference.

\section{Study Design}

A cross sectional randomized, Observational study of Casual comparative design.

\section{Aims and Objectives}

1. Health status of children in remote rural pockets of Himachal Pradesh in and around districts of Solan.

2. Disease profile of children in remote rural pockets of Himachal Pradesh in and around districts of Solan.

3. Anaemia status of children in remote rural pockets of Himachal Pradesh in and around districts of Solan.

\section{Population Sample}

Random selection of health camp for allotment to principal investigator by Head of department for study.

\section{Age Group Studied}

Zero to 18 years.

\section{Inclusion Criteria}

Children attending health camp for paediatrician's consultation.

\section{Exclusion Criteria}

1. Camp held in Urban or semi urban areas.

2. Camp not attended by principal investigator herself.

3. Child attending some other specialty only.

\section{METHODS}

The project was designed to study the clinical profile and health status of children presenting themselves to paediatrician with complaints in health camps held in rural areas of Himachal Pradesh wherever our Institute organized free health camps in collaboration with village representatives. Multispecialty free health camps were conducted by our institute in different urban and rural areas remote rural pockets of Himachal Pradesh in and around Solan, other nearby districts like Sirmour etc. The specialist services in such camps usually included all major branches, specialist from Medicine, Surgery, Orthopaedics, Gynaecologist, Ophthalmologist, Paediatrician including ENT specialist etc. Children in the age group zero to eighteen years attending the Paediatrician in such camps were included in the study. Demography, history details, clinical symptoms, anthropometry and thorough clinical examination was done.
Findings were noted in a predesigned proforma along with provisional diagnosis. Haemoglobin of child was checked on the spot. Those needing further investigations were advised to visit our tertiary care hospital. Those who came, were followed up both for diagnostic investigations and therapeutic interventions. Data was analysed statistically in MS excel, MS access, \& Analyse -it, with descriptive and inferential statistical tests applied as frequencies, percentages, mean, median, mode, SD as applicable to categorical and numerical variables.

Costly investigations like CT/MRI etc. were provided at subsidized rates and routine investigations were done free of cost for these children. Many camps were done by our institute during the study period but only data from those camps which were attended and investigated by the principal investigator and held in remote rural pockets were included in the study.

Material used were usual clinical examination tools. Haemoglobin estimation was done by finger prick method with a conventional haemoglobin meter by laboratory technician present in camp.

\section{RESULTS}

472 children were included in the study through 20 health camps over a period of about two and a half years from July 2016 to March 2019. (Table 1). A total of 6019 patients attended in these 20 camps. Out of these 472 children attended the Paediatrician (7.87\%). Average number of children for paediatric consultation were 23.6 in each camp with median as 23.6 and mode as 27 . Maximum number of patients were in age group 6-12 years forming $41.52 \%$ children, although it includes maximum number of years also, whereas infants were only $2.54 \%$. When children were grouped according to equal number of 6 years assigned i.e. 0 $6,6-12,12-18$ years, then also maximum number of patients were in 6-12 year age group ( $41.52 \%)$, i.e. school going age, $36.86 \%$ patients were of $<6$ years of age and $21.61 \%$ patients belonged to $>12$ years of age.

There were $51.9 \%$ boys and $48.1 \%$ girls. A Sex ratio of 1.07:1 was found, with no statistically significant difference. [Table 2]When number of siblings in family were considered, which may affect the rearing of child, sharing of resources etc. it was found that maximum number of families had a twochild norm (40.68\%). Large families with responsibility for four or more children to support, feed and care were $9.73 \%$.

Health status of children were analysed in terms of their growth parameters as per Indian academy of Paediatrics 2015 growth charts, anaemia status \& overt signs of Vitamin deficiency. $25.6 \%$ children had a weight of $<3$ rd percentile for their age and sex needing immediate and appropriate action while another $23.3 \%$ children had weight $<10^{\text {th }}$ centile, meaning a major chunk of about $48.9 \%$ children were undernourished with moderate to severe PEM. However overweight children were only $0.2 \%$ (Table 3 ). Stature wise $25.4 \%$ had height $<3$ rd percentile and another $13.9 \%$ had height $<10^{\text {th }}$ percentile. Tall stature with Height $>$ 97 percentile was only 1.1\%. Thus, a cumulative $39.3 \%$ children needed an investigative, supportive and periodical follow up approach to achieve their full potential of growth with $25.4 \%$ needing immediate intervention. In $38.1 \%$ children both height and weight were growing parallel to 
each other, while in $36.9 \%$ weight appeared to be affected more and in $25 \%$ height was affected more signifying wasting and growth retardation. (Table 4) As per IAP recommendations, body mass index was not evaluated in $<5$ years old (29.2\%), while percentage of children who were found to be having a BMI of < 5 percentile signalling undernutrition in $>5$ years age group was $12.9 \%$ (Table 5 ). Prevalence of BMI >95 (27 Eq) percentile which is an indicator of overweight \& obesity was only $2.1 \%$, meaning overweight is not a problem in rural Himachal Pradesh while under weight is. Cumulative figure of children with a BMI of $<10^{\text {th }}$ centile is $21.4 \%$ who need a follow up for their growth monitoring.

Apparent pallor was noticed in $30.3 \%$ cases, while haemoglobin estimation revealed high prevalence of anaemia of varying degree in $78.8 \%$ cases as per WHO criteria.[13] Only $21.2 \%$ children had no anaemia. Most of them had mild anaemia (50.4\%), while no case of very severe anaemia was found. (Table 6).Overt signs of Vitamin D deficiency were found in $6.6 \%$ cases. Conjunctival xerosis as a sign of Vitamin A deficiency (WHO Classification X1A) was noted in $2.1 \%$ children and vitamin B complex deficiency was noted in 1.1\% as stomatitis or aphthous ulcers.

A significant number of children were brought with long duration of illness i.e. lasting for more than one year (36.7 \%), while another $5.51 \%$ children were having problems since birth. (Table 7). As per their detailed history and physical examination need for investigations was decided. On analysis it was found that sophisticated and costly tests were needed in many (Chart 1), even chromosomal studies were strongly indicated in 4 cases $(0.85 \%)$ and growth hormone assay in 2 cases. $(0.42 \%)$, although it appeared that few more may require it on follow up. Their previous treatment and investigation history revealed that there were more than $25 \%$ cases at the outset who needed specialist care but only $0.42 \%$ were investigated and were under regular specialist care signalling a big gap between need and supply. This number may further increase after initial investigations.

The most common presenting complaint was pain and the most common site of pain was abdomen. Over all GIT symptoms were predominant, while skin problems also formed a large group. Instead of single complaint multiple complaints dominated. $11.2 \%$ cases presented with growth problems of varying nature and about half of this number $5.5 \%$ presented with developmental delay. Urinary symptoms including bed wetting also formed a large group (Table 8)

On basis of history, thorough clinical examination, anthropometric measurements and haemoglobin estimation, a provisional diagnosis was made. Patients and parents were counselled for investigations on case to case basis and final diagnosis was made on follow up if they came for investigations. Those who did not, their initial provisional diagnosis was considered final. Clinical Disease burden was stratified as per International classification of diseases (ICD 10-2018) divided in 21 chapters, as per definitions in classification of disease manual and meeting the criteria. It indicated that anaemia and nutrition i.e. chapter 3, 4 formed the bulk of disease burden, while 5 chapters were marked by their absence, like neoplasms etc. (Table 9)

\begin{tabular}{|c|c|c|c|c|c|c|c|c|c|}
\hline \multirow{2}{*}{$\begin{array}{l}\dot{j} \\
\dot{j}\end{array}$} & \multirow{2}{*}{ 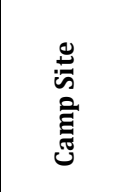 } & \multirow{2}{*}{ 苛 } & \multirow{2}{*}{ 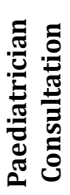 } & \multicolumn{6}{|c|}{$\begin{array}{c}\text { Age Wise Distribution } \\
\text { (Years) }\end{array}$} \\
\hline & & & & $0-1$ & $1-3$ & 3-6 & $6-12$ & $12-14$ & $14-18$ \\
\hline 1 & Joharji & 146 & 14 & 2 & 2 & 3 & 4 & 2 & 1 \\
\hline 2 & Sapooran & 270 & 19 & 1 & 4 & 8 & 6 & 0 & 0 \\
\hline 3 & Gaggal & 280 & 17 & 0 & 1 & 4 & 8 & 4 & 0 \\
\hline 4 & Balera & 280 & 6 & 0 & 0 & 0 & 3 & 3 & 0 \\
\hline 5 & Nohradhar & 260 & 9 & 0 & 4 & 0 & 2 & 2 & 1 \\
\hline 6 & \begin{tabular}{|l|} 
Padhog \\
\end{tabular} & 290 & 30 & 2 & 4 & 7 & 9 & 4 & 4 \\
\hline 7 & Salogra & 450 & 39 & 0 & 4 & 8 & 14 & 8 & 5 \\
\hline 8 & Bhogdhar & 180 & 31 & 0 & 9 & 8 & 10 & 3 & 1 \\
\hline 9 & Dadahu, & 680 & 26 & 0 & 2 & 7 & 10 & 3 & 4 \\
\hline 10 & Sadopul & 268 & 27 & 1 & 3 & 3 & 16 & 4 & 0 \\
\hline 11 & Jadala & 120 & 15 & 1 & 4 & 3 & 6 & 1 & 0 \\
\hline 12 & Koti & 590 & 40 & 2 & 5 & 12 & 14 & 4 & 3 \\
\hline 13 & Dumehar & 295 & 29 & 1 & 3 & 6 & 13 & 4 & 2 \\
\hline 14 & Giripul & 140 & 14 & 0 & 0 & 2 & 8 & 2 & 2 \\
\hline 15 & Maanju & 300 & 29 & 1 & 3 & 6 & 13 & 4 & 2 \\
\hline 16 & Chamiya & 340 & 27 & 0 & 4 & 7 & 12 & 3 & 1 \\
\hline 17 & Banethi & 350 & 28 & 0 & 2 & 3 & 15 & 6 & 2 \\
\hline 18 & Arki & 280 & 26 & 1 & 3 & 5 & 12 & 4 & 1 \\
\hline 19 & Kathod & 340 & 27 & 0 & 3 & 1 & 15 & 6 & 2 \\
\hline 20 & Dimber & 160 & 19 & 0 & 4 & 5 & 6 & 2 & 2 \\
\hline & \begin{tabular}{|l|} 
Total \\
\end{tabular} & 6019 & 472 & 12 & 64 & \begin{tabular}{|l|}
98 \\
\end{tabular} & 196 & 69 & 33 \\
\hline & Percentage & $\%$ & 7.84 & \begin{tabular}{|l|}
2.54 \\
\end{tabular} & 13.56 & 20.76 & 41.52 & 14.62 & 6.99 \\
\hline \multicolumn{10}{|c|}{ Table 1. Age Wise Distribution of Patients } \\
\hline
\end{tabular}

\begin{tabular}{|c|c|c|c|c|c|c|c|c|c|c|}
\hline \multirow{2}{*}{$\begin{array}{c}\text { Sl. } \\
\text { No. }\end{array}$} & \multirow{2}{*}{$\begin{array}{l}\text { Camp } \\
\text { Site }\end{array}$} & \multirow{2}{*}{$\begin{array}{c}\text { No. } \\
\text { of } \\
\text { Children }\end{array}$} & \multirow{2}{*}{\multicolumn{2}{|c|}{\begin{tabular}{|c|}
$\begin{array}{c}\text { Sex Wise } \\
\text { Distribution }\end{array}$ \\
Male
\end{tabular}}} & \multicolumn{6}{|c|}{$\begin{array}{c}\begin{array}{c}\text { Number of Siblings Wise } \\
\text { (Family Size) }\end{array} \\
\end{array}$} \\
\hline & & & & & \begin{tabular}{l|l}
0 \\
\end{tabular} & 1 & 2 & 3 & & $5 />$ \\
\hline 1 & Joharji & 14 & 9 & 5 & 4 & 6 & 3 & 1 & 0 & 0 \\
\hline 2 & Sapooran & 19 & 13 & 6 & 1 & 11 & 6 & 1 & 0 & 0 \\
\hline 3 & Gaggal & 17 & 8 & 9 & 5 & 10 & 2 & 0 & 0 & 0 \\
\hline 4 & Balera & 6 & 5 & 1 & 2 & 3 & 1 & 0 & 0 & 0 \\
\hline 5 & Nohradhar & 9 & 5 & 4 & 4 & 3 & 1 & 0 & 1 & 0 \\
\hline 6 & Padhog & 30 & 15 & 15 & 6 & 15 & 4 & 5 & 0 & 0 \\
\hline 7 & Salogra & 39 & 21 & 18 & 14 & 16 & 7 & 2 & 0 & 0 \\
\hline 8 & Bhogdhar & 31 & 13 & 18 & 15 & 11 & 4 & 1 & 0 & 0 \\
\hline 9 & Dadahu, & 26 & 15 & 11 & 7 & 7 & 7 & 3 & 2 & 0 \\
\hline 10 & Sadopul & 27 & 13 & 14 & 9 & 8 & 4 & 5 & 0 & 1 \\
\hline 11 & Jadala & 15 & 7 & 8 & 5 & 6 & 3 & 1 & 0 & 0 \\
\hline 12 & Koti & 40 & 25 & 15 & 12 & 16 & 7 & 3 & 2 & 0 \\
\hline 13 & Dumehar & 29 & 13 & 16 & 9 & 12 & 5 & 2 & 0 & 1 \\
\hline 14 & Giripul & 14 & 3 & 11 & 4 & 6 & 3 & 1 & 0 & 0 \\
\hline 15 & Maanju & 29 & 15 & 14 & 9 & 12 & 6 & 2 & 0 & 0 \\
\hline 16 & Chamiya & 27 & 14 & 13 & 8 & 11 & 6 & 1 & 0 & 1 \\
\hline 17 & Banethi & 28 & 13 & 15 & 9 & 13 & 5 & 0 & 1 & 0 \\
\hline 18 & Arki & 26 & 14 & 12 & 8 & 10 & 5 & 3 & 0 & 0 \\
\hline 19 & Kathod & 27 & 13 & 14 & 6 & 10 & 8 & 3 & 0 & 0 \\
\hline \multirow[t]{3}{*}{20} & Dimber & 19 & 11 & 8 & 5 & 6 & 5 & 1 & 1 & 1 \\
\hline & Total & 472 & 245 & 227 & 142 & 192 & 92 & 35 & 7 & 4 \\
\hline & Percentage & 7.84 & 51.9 & 48.1 & 30.08 & \begin{tabular}{|l|}
40.68 \\
\end{tabular} & 19.49 & 7.41 & 1.48 & 0.84 \\
\hline
\end{tabular}

\begin{tabular}{|c|c|c|c|c|c|c|c|c|c|c|}
\hline \multirow[b]{2}{*}{$\begin{array}{l}\text { Sl. } \\
\text { No. }\end{array}$} & \multirow[b]{2}{*}{$\begin{array}{l}\text { Camp } \\
\text { Site }\end{array}$} & \multirow[b]{2}{*}{\begin{tabular}{|c} 
No. of \\
Children
\end{tabular}} & \multicolumn{8}{|c|}{ Weight Percentiles } \\
\hline & & & $\begin{array}{l}<3 \\
\text { or } 3\end{array}$ & \begin{tabular}{|l|}
$<10$ \\
or 10
\end{tabular} & $\begin{array}{l}<25 \\
\text { or } 25\end{array}$ & $\begin{array}{l}<50 \\
\text { or } 50\end{array}$ & $\begin{array}{c}<75 \\
0 \text { or } 75\end{array}$ & $\begin{array}{l}<90 \\
\text { or } 90\end{array}$ & \begin{tabular}{|c|}
$<97$ \\
or 97
\end{tabular} & $7>97$ \\
\hline 1 & Joharji & 14 & 3 & 4 & 5 & 2 & 0 & 0 & 0 & 0 \\
\hline 2 & Sapooran & & 6 & 4 & 6 & 3 & 0 & 0 & 0 & 0 \\
\hline 3 & & 1 & 4 & 3 & 7 & 3 & ( & 0 & 0 & 0 \\
\hline 4 & & & 1 & 3 & 1 & 0 & & & 0 & 0 \\
\hline 5 & Nohradhar & & 4 & 2 & 1 & 2 & ( & 0 & 0 & 0 \\
\hline 6 & & 30 & 6 & 7 & 6 & 2 & 3 & 4 & 2 & 0 \\
\hline 7 & Salogra & & 8 & 9 & 5 & 8 & & & 3 & 1 \\
\hline 8 & Bhogdhar & & 12 & 9 & 4 & 3 & & 0 & 0 & 0 \\
\hline 9 & Dadah & & 22 & 6 & 10 & 3 & 5 & 0 & 0 & 0 \\
\hline 10 & & & 5 & 5 & 12 & 4 & 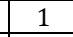 & 0 & 0 & 0 \\
\hline 11 & Jadala & & 7 & 1 & 4 & 1 & & 0 & 1 & 0 \\
\hline 12 & Koti & 40 & 15 & 8 & 8 & 7 & 2 & 0 & 0 & 0 \\
\hline 13 & Dumehar & 2 & 7 & 6 & 2 & 5 & 8 & 1 & 0 & 0 \\
\hline 14 & & & 6 & 2 & 0 & 3 & & & 0 & 0 \\
\hline 15 & & & 6 & 8 & 5 & 6 & 4 & 0 & 0 & 0 \\
\hline 16 & Chamiya & 27 & 9 & 4 & 5 & 2 & 6 & 1 & 0 & 0 \\
\hline 17 & Banethi & 28 & 8 & 6 & 10 & 3 & 0 & 0 & 1 & 0 \\
\hline
\end{tabular}




\begin{tabular}{|c|c|c|c|c|c|c|c|c|c|c|}
\hline 18 & Arki & 26 & 3 & 9 & 7 & 5 & 1 & 1 & 0 & 0 \\
\hline 19 & Kathod & 27 & 4 & 9 & 5 & 5 & 4 & 0 & 0 & 0 \\
\hline 20 & Dimber & 19 & 5 & 5 & 7 & 0 & 2 & 0 & 0 & 0 \\
\hline & Total & $\mathbf{4 7 2}$ & $\mathbf{1 2 1}$ & $\mathbf{1 1 0}$ & $\mathbf{1 1 0}$ & $\mathbf{6 7}$ & $\mathbf{4 7}$ & $\mathbf{9}$ & $\mathbf{7}$ & $\mathbf{1}$ \\
\hline & Percentage & $\%$ & 25.6 & 23.3 & 23.3 & 14.2 & 9.9 & 1.9 & 1.5 & 0.2 \\
\hline \multicolumn{7}{|c|}{ Table 3. Weight Percentiles of Children in Rural Pockets } \\
\hline
\end{tabular}

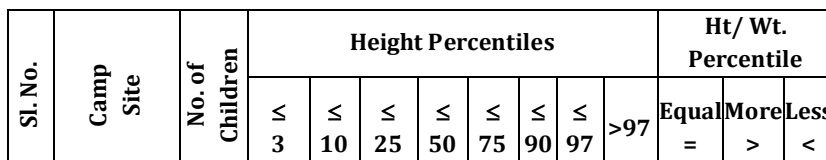

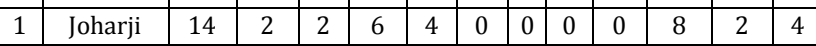

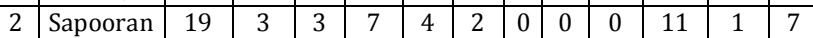
\begin{tabular}{|l|l|l|l|l|l|l|l|l|l|l|l|l|l|}
\hline 3 & Gaggal & 17 & 7 & 2 & 4 & 4 & 0 & 0 & 0 & 0 & 8 & 4 & 5 \\
\hline
\end{tabular} \begin{tabular}{l|l|l|l|l|l|l|l|l|l|l|l|l|l|}
4 & Balera & 6 & 1 & 1 & 1 & 2 & 1 & 0 & 0 & 0 & 2 & 1 & 3 \\
\hline
\end{tabular} \begin{tabular}{|lll|lllllllllllllllll}
5 & Nohradhar & 9 & 2 & 2 & 1 & 2 & 0 & 0 & 2 & 0 & 4 & 0 & 5 \\
\hline
\end{tabular} \begin{tabular}{|c|c|c|c|c|c|c|c|c|c|c|c|c|c|}
\hline 6 & Padhog & 30 & 5 & 3 & 9 & 2 & 6 & 4 & 1 & 0 & 8 & 10 & 12 \\
\hline 7 & Salo & 39 & 9 & 5 & 8 & 8 & 4 & 1 & 2 & 2 & 9 & 14 & 16 \\
\hline
\end{tabular} \begin{tabular}{|l|l|l|l|l|l|l|l|l|l|l|l|l|l|}
7 & Salogra & 39 & 9 & 5 & 8 & 8 & 4 & 1 & 2 & 2 & 9 & 14 & 16 \\
\hline
\end{tabular} \begin{tabular}{|c|l|l|l|l|l|l|l|l|l|l|l|l|l|}
\hline 8 & Bhogdhar & 31 & 15 & 5 & 6 & 0 & 3 & 1 & 1 & 0 & 15 & 8 & 8 \\
\hline
\end{tabular} \begin{tabular}{|l|l|l|l|l|l|l|l|l|l|l|l|l|l|}
\hline 9 & Dadahu & 26 & 12 & 6 & 0 & 4 & 1 & 2 & 0 & 1 & 6 & 17 & 3 \\
\hline
\end{tabular}

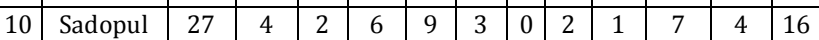

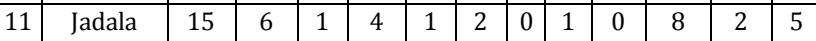
\begin{tabular}{|l|l|l|l|l|l|l|l|l|l|l|l|l|l|}
12 & Koti & 40 & 11 & 5 & 8 & 10 & 2 & 4 & 0 & 0 & 18 & 3 & 19 \\
\hline
\end{tabular}

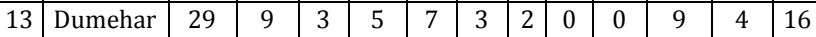
\begin{tabular}{|l|l|l|l|l|l|l|l|l|l|l|l|l|l|}
14 & Giripul & 14 & 4 & 3 & 3 & 3 & 1 & 0 & 0 & 0 & 7 & 4 & 3 \\
\hline
\end{tabular}

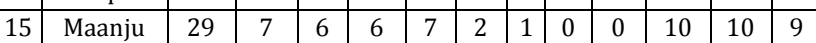
\begin{tabular}{|l|l|l|l|l|l|l|l|l|l|l|l|l|l|}
16 & Chamiya & 27 & 4 & 2 & 5 & 6 & 8 & 1 & 1 & 0 & 12 & 7 & 8 \\
\hline
\end{tabular} \begin{tabular}{|l|l|l|l|l|l|l|l|l|l|l|l|l|l|}
17 & Banethi & 28 & 5 & 3 & 6 & 4 & 7 & 2 & 1 & 0 & 11 & 8 & 9 \\
\hline
\end{tabular} \begin{tabular}{l|l|l|l|l|l|l|l|l|l|l|l|l|l|}
18 & Arki & 26 & 6 & 2 & 4 & 8 & 6 & 0 & 0 & 0 & 6 & 10 & 10 \\
\hline
\end{tabular} \begin{tabular}{|l|l|l|l|l|l|l|l|l|l|l|l|l|l|}
19 & Kathod & 27 & 5 & 7 & 2 & 7 & 3 & 2 & 0 & 1 & 12 & 7 & 8 \\
\hline
\end{tabular}

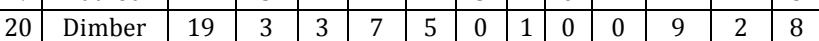
\begin{tabular}{|l|c|c|c|c|c|c|c|c|c|c|c|c|} 
Total & 472 & 120 & 66 & 98 & 97 & 54 & 21 & 11 & 5 & 180 & 118 & 174 \\
\hline
\end{tabular} \begin{tabular}{|l|c|c|c|c|c|c|c|c|c|c|c|c|}
\hline Percentage & $\%$ & 25.4 & 13.9 & 20.8 & 20.6 & 11.4 & 4.4 & 2.3 & 1.1 & 38.1 & 25.0 & 36.9 \\
\hline
\end{tabular} Table 4. Height Percentiles of Children and Their Relationship with Weight Percentile

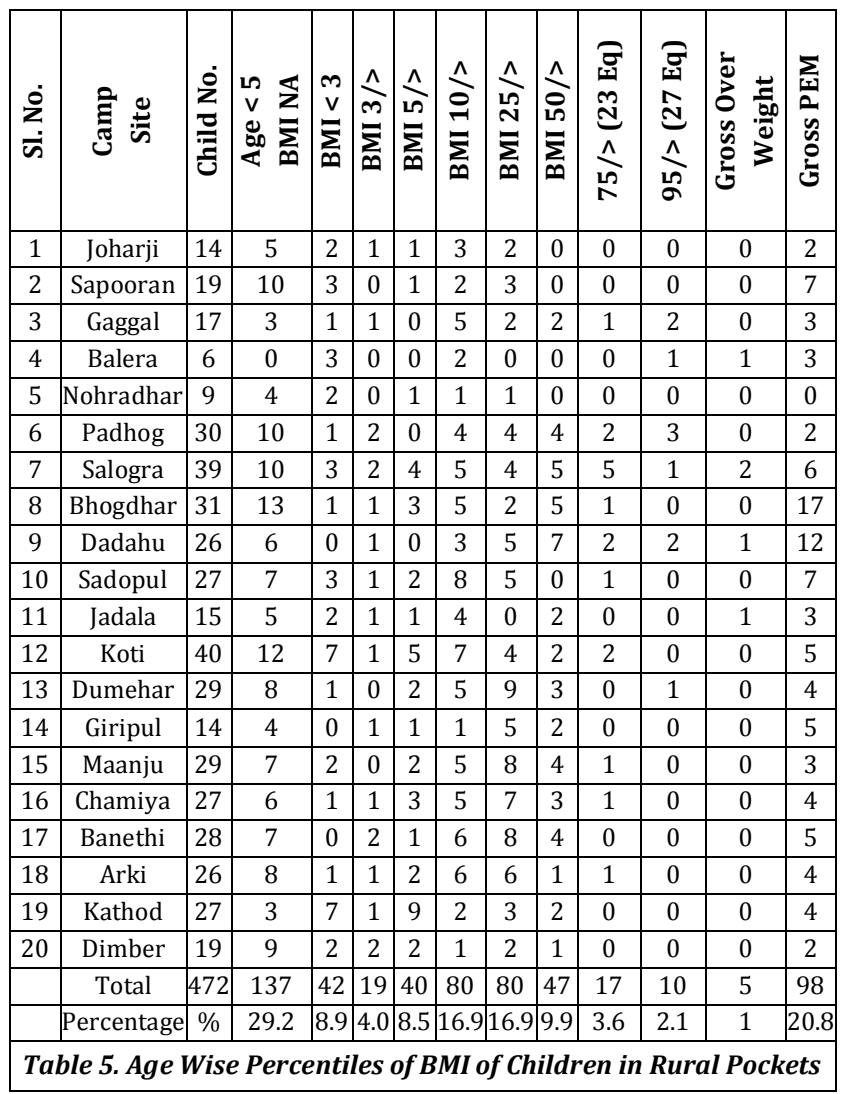

\begin{tabular}{|c|c|c|c|c|c|c|c|c|c|c|c|}
\hline \multirow[b]{2}{*}{$\begin{array}{l}\dot{0} \\
\dot{n} \\
\dot{n}\end{array}$} & \multirow[b]{2}{*}{ 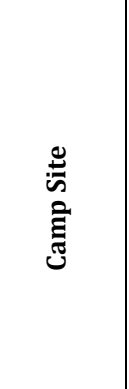 } & \multirow[b]{2}{*}{ 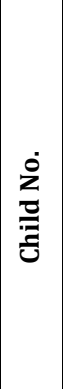 } & \multirow[b]{2}{*}{ 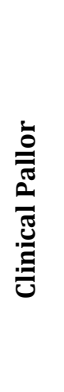 } & \multicolumn{5}{|c|}{$\begin{array}{c}\text { Age Wise } \\
\text { Haematological } \\
\text { Grading }\end{array}$} & \multicolumn{3}{|c|}{$\begin{array}{c}\text { Clinical } \\
\text { Vitamin } \\
\text { Deficiency }\end{array}$} \\
\hline & & & & 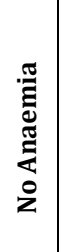 & 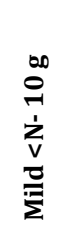 & 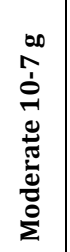 & $\begin{array}{l}\infty \\
\hat{v} \\
0 \\
\dot{\omega} \\
\grave{d} \\
\omega\end{array}$ & 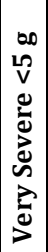 & $\stackrel{P}{\prime}$ & $\stackrel{\square}{ز}$ & 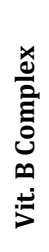 \\
\hline 1 & Joharji & 14 & 5 & 4 & 7 & 2 & 1 & 0 & 2 & 0 & 0 \\
\hline 2 & Sapooran & 19 & 7 & 5 & 10 & 4 & 0 & 0 & 5 & 0 & 1 \\
\hline 3 & Gaggal & 17 & 3 & 2 & 8 & 6 & 1 & 0 & 1 & 1 & 0 \\
\hline 4 & Balera & 6 & 1 & 1 & 4 & 1 & 0 & 0 & 0 & 0 & 0 \\
\hline 51 & Nohradhar & 9 & 4 & 0 & 3 & 4 & 2 & 0 & 0 & 2 & 0 \\
\hline \begin{tabular}{l|l}
6 \\
\end{tabular} & Padhog & 30 & 10 & 5 & 17 & 8 & 0 & 0 & 1 & 0 & 0 \\
\hline 7 & Salogra & 39 & 9 & 14 & 20 & 5 & 0 & 0 & 1 & 0 & 1 \\
\hline 8 & Bhogdhar & 31 & 8 & 8 & 18 & 5 & 0 & 0 & 3 & 1 & 0 \\
\hline \begin{tabular}{l|l}
9 \\
\end{tabular} & Dadahu & 26 & 8 & 5 & 13 & 7 & 1 & 0 & 3 & 0 & 0 \\
\hline 10 & Sadopul & 27 & 5 & 0 & 8 & 19 & 0 & 0 & 2 & 0 & 0 \\
\hline 11 & Jadala & 15 & 5 & 3 & 7 & 4 & 1 & 0 & 1 & 0 & 0 \\
\hline 12 & Koti & 40 & 15 & 8 & 20 & 10 & 2 & 0 & 1 & 0 & 1 \\
\hline 13 & Dumehar & 29 & 11 & 6 & 15 & 7 & 1 & 0 & 1 & 0 & 0 \\
\hline 14 & Giripul & 14 & 6 & 3 & 7 & 4 & 0 & 0 & 1 & 0 & 1 \\
\hline 15 & Maanju & 29 & 12 & 5 & 15 & 8 & 1 & 0 & 1 & 1 & 0 \\
\hline 16 & Chamiya & 27 & 7 & 7 & 14 & 6 & 0 & 0 & 0 & 0 & 0 \\
\hline \begin{tabular}{|l|}
17 \\
\end{tabular} & Banethi & 28 & 9 & 7 & 16 & 5 & 0 & 0 & 2 & 1 & 0 \\
\hline \begin{tabular}{|l|}
18 \\
\end{tabular} & Arki & 26 & 8 & 2 & 17 & 6 & 1 & 0 & 1 & 0 & 0 \\
\hline \begin{tabular}{|l|}
19 \\
\end{tabular} & Kathod & 27 & 6 & 5 & 12 & 10 & 0 & 0 & 3 & 3 & 1 \\
\hline 20 & Dimber & 19 & 4 & 10 & 7 & 2 & 0 & 0 & 2 & 1 & 0 \\
\hline & Total & 472 & 143 & 100 & 238 & 123 & 11 & $\mathbf{0}$ & 31 & 10 & 5 \\
\hline & Percentage & $\%$ & 30.3 & 21.2 & 50.4 & 26.0 & 2.3 & 0 & 6.6 & 2.1 & 1.1 \\
\hline
\end{tabular}

\begin{tabular}{|c|c|c|c|c|c|c|c|c|c|c|c|}
\hline $\begin{array}{l}\dot{0} \\
\dot{z} \\
\dot{\omega}\end{array}$ & $\begin{array}{l}\stackrel{\Xi}{5} \\
\text { है } \\
\text { तु }\end{array}$ & 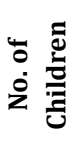 & $\begin{array}{l}\tilde{z} \\
\hat{\tilde{\tau}} \\
\tilde{\tilde{v}} \\
\tilde{v}\end{array}$ & 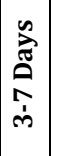 & 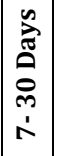 & 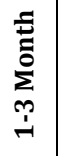 & $\begin{array}{l}\tilde{I} \\
\vdots \\
\sum_{0} \\
\dot{D} \\
\dot{n}\end{array}$ & $\begin{array}{ll}1 & \\
\vdots & 0 \\
0 & 0\end{array}$ & $\stackrel{\sim}{\stackrel{\Xi}{\Xi}}$ & $\wedge \underset{\beth}{~}$ & 芦苛 \\
\hline 1 & Joharji & 14 & 0 & 2 & 2 & 0 & 1 & 5 & 2 & 2 & 0 \\
\hline 2 & Sapooran & 19 & 1 & 2 & 2 & 4 & 1 & 4 & 1 & 4 & 0 \\
\hline 3 & Gaggal & 17 & 0 & 1 & 0 & 1 & 1 & 2 & 4 & 8 & 0 \\
\hline 4 & Balera & 6 & 0 & 0 & 0 & 0 & 1 & 1 & 2 & 1 & 1 \\
\hline 5 & Nohradhar & 9 & 0 & 0 & 0 & 0 & 0 & 2 & 1 & 4 & 2 \\
\hline 6 & Padhog & 30 & 1 & 2 & 3 & 5 & 4 & 7 & 2 & 4 & 2 \\
\hline 7 & Salogra & 39 & 3 & 4 & 4 & 3 & 7 & 4 & 6 & 6 & 2 \\
\hline 8 & Bhogdhar & 31 & 1 & 1 & 3 & 2 & 6 & 6 & 4 & 5 & 3 \\
\hline 9 & \begin{tabular}{|l|} 
Dadahu \\
\end{tabular} & 26 & 1 & 2 & 0 & 1 & 3 & 6 & 7 & 4 & 2 \\
\hline 10 & Sadopul & 27 & 0 & 1 & 1 & 5 & 4 & 4 & 6 & 4 & 2 \\
\hline 11 & Jadala & 15 & 0 & 1 & 1 & 1 & 2 & 4 & 3 & 3 & 0 \\
\hline 12 & Koti & 40 & 1 & 2 & 3 & 4 & 5 & 8 & 7 & 8 & 2 \\
\hline 13 & Dumehar & 29 & 1 & 1 & 3 & 4 & 4 & 5 & 5 & 5 & 1 \\
\hline 14 & Giripul & 14 & 0 & 0 & 0 & 2 & 1 & 1 & 2 & 5 & 3 \\
\hline 15 & Maanju & 29 & 1 & 2 & 3 & 3 & 4 & 5 & 6 & 4 & 1 \\
\hline 16 & \begin{tabular}{|l|} 
Chamiya \\
\end{tabular} & 27 & 0 & 1 & 2 & 3 & 3 & 7 & 6 & 4 & 1 \\
\hline 17 & Banethi & 28 & 1 & 0 & 2 & 2 & 5 & 6 & 5 & 7 & 0 \\
\hline 18 & Arki & 26 & 1 & 0 & 1 & 4 & 4 & 5 & 6 & 5 & 0 \\
\hline 19 & Kathod & 27 & 1 & 2 & 2 & 4 & 4 & 2 & 6 & 4 & 2 \\
\hline \multirow[t]{3}{*}{20} & Dimber & 19 & 0 & 0 & 5 & 4 & 2 & 1 & 3 & 2 & 2 \\
\hline & Total & 472 & 13 & 24 & 37 & 52 & 62 & 85 & 84 & 89 & 26 \\
\hline & Percentage & $\%$ & 2.75 & $5.087^{7}$ & 7.84 & 11.0 & 13.13 & 318.0 & \begin{tabular}{|l|l|}
17.79 \\
\end{tabular} & \begin{tabular}{|l|l|}
18.9 \\
\end{tabular} & 5.51 \\
\hline
\end{tabular}




\begin{tabular}{|c|c|}
\hline Complaint & No. \\
\hline $\begin{array}{c}\text { Pain - Abdomen 62/ Head 10/Chest 7/Limbs 20/ Back } 3 / \\
\text { Earache 4/ Throat pain } 6\end{array}$ & 112 \\
\hline $\begin{array}{c}\text { GI: Vomiting 4/Acidity 4/ Regurgitation 2/ Diarrhoea 19/ Flatulence } \\
\text { 2/ Bleeding PR 3/ Constipation 10/ Worm infestation } 25\end{array}$ & 69 \\
\hline Eating Problems: Poor appetite 26/Overeating 1 /eating inedible 23 & 50 \\
\hline Cough 30/ Running nose 22 & 52 \\
\hline $\begin{array}{c}\text { Respiratory problem: Breathlessness 14/ Mouth breathing 6/ } \\
\text { Wheezing 7/ Throat swelling } 16\end{array}$ & 43 \\
\hline $\begin{array}{l}\text { Skin problem: Rash 10/ Allergy 13/ Hypo pigmented patch 14/Warts } \\
\text { 8/ lesion 4/ itching 7/Acne 2/ furuncles } 1\end{array}$ & 59 \\
\hline $\begin{array}{c}\text { Fever }<7 \text { days: } 31 / \text { Prolonged fever } 6 \text { / Long duration fever }>30 \text { days: } \\
\text { None }\end{array}$ & 37 \\
\hline $\begin{array}{c}\text { Growth Problem: Not gaining weight } 27 / \text { not gaining Height 13/ } \\
\text { Failure to thrive } 11 \text { / Overweight } 4\end{array}$ & 53 \\
\hline $\begin{array}{c}\text { Urinary problem:/ Bed Wetting 10/ Burning micturition 14/ KUB } \\
\text { Stone 1/ Polyuria } 3\end{array}$ & 28 \\
\hline $\begin{array}{c}\text { Developmental problem: Delayed Development 5/ Mental } \\
\text { retardation 4/ Learning 5/ Behavioural } 12\end{array}$ & 26 \\
\hline $\begin{array}{l}\text { Eye Problem: Vision 10/Squint 3/ Conjunctivitis 5/ Stye 1/ cataract } \\
1 \text { / Conjunctival haemorrhage 1/Eye discharge since birth } 1\end{array}$ & 22 \\
\hline Weakness 26 & 26 \\
\hline Swelling or lump 15 / post burn stricture 1 & 16 \\
\hline $\begin{array}{l}\text { Ear problem: hearing difficulty } 3 / \text { ear discharge } 10 / \text { WAX } 2 \text { /external } \\
\text { ear lesion } 1\end{array}$ & 16 \\
\hline Dental problem: Caries 10/ Delayed dentition 3 & 13 \\
\hline $\begin{array}{l}\text { Hair problem: loss of hair } 1 / \text { dandruff } 5 / \text { Thin hair } 1 / \\
\text { Scalp lesions } 1\end{array}$ & 8 \\
\hline Menstrual problem: Excessive 5/ irregular 1/Vaginal discharge 1 & 7 \\
\hline Speech problem: Delayed 2/ Stamm & 9 \\
\hline High Blood pressure 1/ Heart problem 2/ Fainting 2 & 5 \\
\hline Jaundice 3 & 3 \\
\hline $\begin{array}{c}\text { Others: Congenital problems 9/ Nail discoloration 2/Seizures } \\
\text { 2/Diabetes 2/breast swelling } 1 \text { etc. }\end{array}$ & 16 \\
\hline \multicolumn{2}{|c|}{ Table 8. Presenting Complaints with Frequen } \\
\hline
\end{tabular}

\begin{tabular}{|c|c|c|c|}
\hline $\begin{array}{l}\text { ICD } 10 \\
\text { Chapter }\end{array}$ & Description & Frequency & $\%$ \\
\hline 1 & Certain infections and parasitic diseases & 51 & 10.81 \\
\hline 2 & \begin{tabular}{|c|} 
Neoplasms \\
\end{tabular} & 0 & \begin{tabular}{|l|}
0 \\
\end{tabular} \\
\hline 3 & $\begin{array}{l}\text { Diseases of blood and blood forming } \\
\text { organs and certain disorders involving } \\
\text { the immune mechanism }\end{array}$ & 372 & 78.81 \\
\hline 4 & $\begin{array}{l}\text { Endocrine, nutritional and metabolic } \\
\text { diseases }\end{array}$ & 277 & 58.68 \\
\hline 5 & $\begin{array}{c}\text { Mental, Behavioural and } \\
\text { neurodevelopmental disorders }\end{array}$ & 35 & 7.41 \\
\hline 6 & Diseases of the nervous system & 5 & 1.06 \\
\hline 7 & Diseases of the eye and adnexa & 22 & 4.66 \\
\hline 8 & Diseases of the ear and mastoid process & 26 & 5.50 \\
\hline 9 & Diseases of the circulatory system & 1 & 0.21 \\
\hline 10 & Diseases of the respiratory system & 43 & 9.11 \\
\hline 11 & Diseases of the digestive system & 82 & 17.37 \\
\hline 12 & $\begin{array}{c}\text { Diseases of the skin and subcutaneous } \\
\text { tissue }\end{array}$ & 43 & 9.11 \\
\hline 13 & $\begin{array}{c}\text { Diseases of the musculoskeletal system } \\
\text { and connective tissue }\end{array}$ & 1 & 0.21 \\
\hline 14 & Diseases of the genitourinary system & 31 & 6.56 \\
\hline 15 & $\begin{array}{l}\text { Pregnancy, child birth and the } \\
\text { puerperium }\end{array}$ & 0 & 0 \\
\hline 16 & \begin{tabular}{|c|} 
certain conditions originating in \\
perinatal period
\end{tabular} & 5 & 1.06 \\
\hline 17 & $\begin{array}{c}\text { Congenital malformations, deformations } \\
\text { and chromosomal abnormalities }\end{array}$ & 15 & 3.17 \\
\hline 18 & $\begin{array}{c}\text { Symptoms signs and abnormal clinical } \\
\text { and laboratory findings, not elsewhere } \\
\text { classified }\end{array}$ & 0 & 0 \\
\hline 19 & $\begin{array}{c}\text { Injury, poisoning and certain other } \\
\text { consequences of external causes }\end{array}$ & 2 & 0.42 \\
\hline 20 & External causes of morbidity & 0 & 0 \\
\hline 21 & $\begin{array}{l}\text { Factors influencing health status and } \\
\text { contact with health services }\end{array}$ & 0 & 0 \\
\hline \multicolumn{4}{|c|}{ Table 9. Disease Burden as per ICD 10} \\
\hline
\end{tabular}

\begin{tabular}{|c|c|c|}
\hline Diagnosis & No. & $\begin{array}{c}\text { \% of Total Children } \\
\text { (Out of 472 ) }\end{array}$ \\
\hline Down Syndrome & 2 & $0.42 \%$ \\
\hline Sturge weber Syndrome & 1 & $0.21 \%$ \\
\hline Cavernous haemangioma & 1 & $0.21 \%$ \\
\hline Capillary haemangioma & 1 & $0.21 \%$ \\
\hline Ataxia telangiectasia with Learning \\
disability & 1 & $0.21 \%$ \\
\hline Laryngomalacia & 1 & $0.21 \%$ \\
\hline Dysmorphism & 4 & $0.85 \%$ \\
\hline Global developmental Delay & 5 & $1.05 \%$ \\
\hline Growth Hormone deficiency & 2 & $0.42 \%$ \\
\hline Congenital Cataract/ Congenital rubella \\
syndrome & 1 & $0.21 \%$ \\
\hline Ventricular Septal Defect & 2 & $0.42 \%$ \\
\hline $\begin{array}{c}\text { Posterior urethral valve with Chronic } \\
\text { Renal Failure }\end{array}$ & 1 & $0.21 \%$ \\
\hline Cerebral Palsy & 2 & $0.42 \%$ \\
\hline Microcephaly & 1 & $0.21 \%$ \\
\hline Nephrotic syndrome & 2 & $0.42 \%$ \\
\hline Hydrocephalus & 2 & $0.42 \%$ \\
\hline Typical butter fly rash of TS/ SLE & 2 & $0.42 \%$ \\
\hline Middle cerebral artery thrombosis with \\
hemiparesis & 1 & $0.21 \%$ \\
\hline Tubercular sinus & 1 & $0.21 \%$ \\
\hline Preauricular sinus & 1 & $0.21 \%$ \\
\hline Dost Injection persistent Neuralgia & 1 & $0.21 \%$ \\
\hline Neurocysticercosis & 2 & $0.42 \%$ \\
\hline TotaL (8.05\% of 472) & 1 & $0.21 \%$ \\
\hline Congenital/Genetic/Rare or Uncommon Diseases Found \\
\hline
\end{tabular}
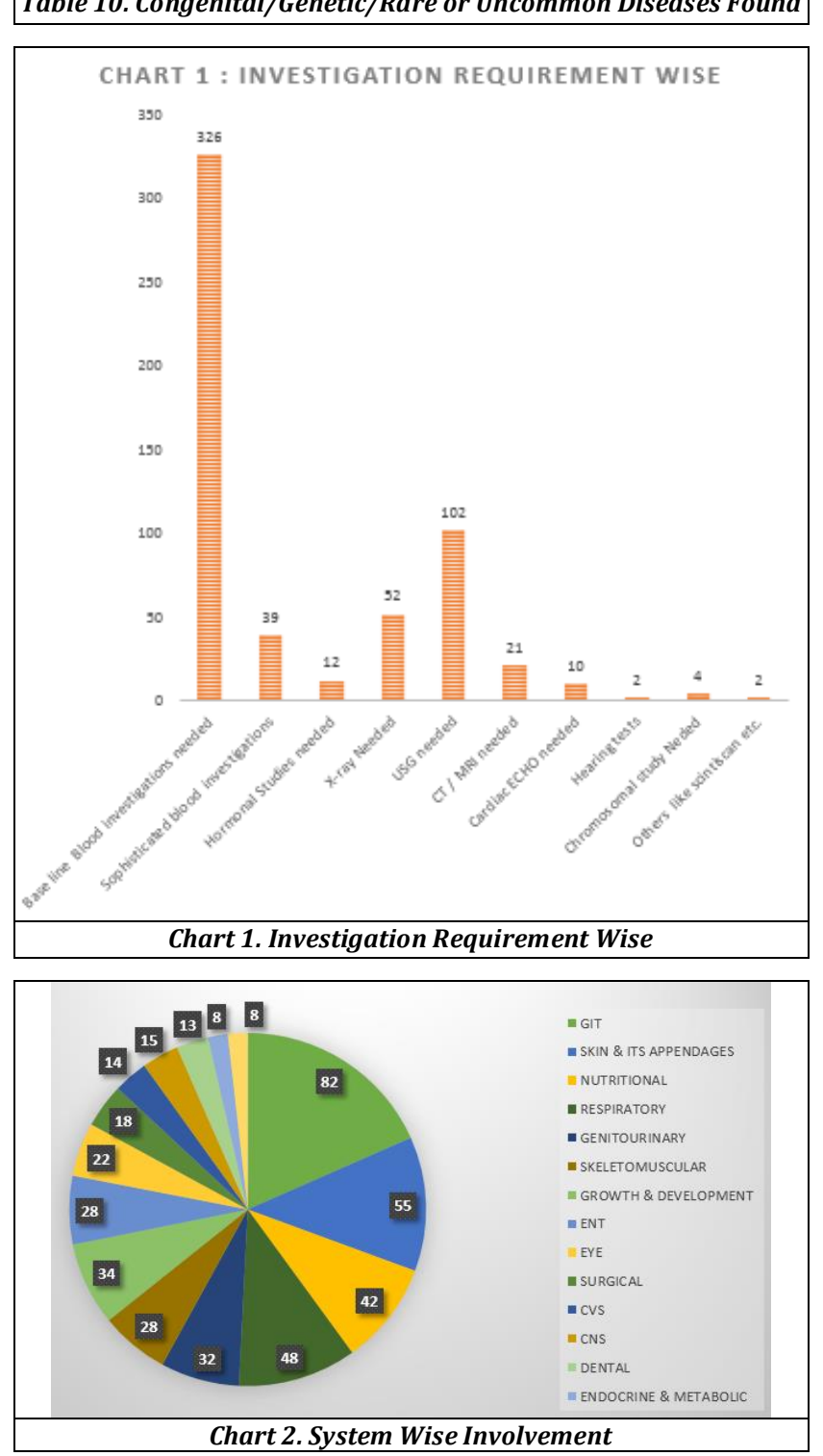
Physical examination focused on system involvement revealed; gastrointestinal tract as the most commonly involved system, followed by Skin \& its appendages. Respiratory system \& Genito urinary system followed closely, Nutritional problems, along with growth and development involvement also formed a significant number. (Chart 2)

As per disease prevalence, most of the cases had multiple problems. Anaemia and PEM were pre-existing or precipitating factors. Growth \& developmental problems with varying grade of severity existed. Short stature was found in $3.2 \%$ cases, Failure to thrive in $2.54 \%$ while Global developmental delay was noted in $1.8 \%$ cases. Communicable diseases and bacterial infections were less common. Behavioural problems formed a significant number, Nocturnal enuresis above the age of 5 was present in $2.3 \%$ and PICA was noted in $5.5 \%$ cases. Skin problems were present in form of Pityriasis versicolor, tinea corporis and dermatitis mainly. Diagnosis and description of each individual case is beyond the scope of the paper.

The important and unexpected observation was the presence of uncommon \& rare diseases, Syndromes, CNS diseases which formed a significant part of spectrum of disease pattern. (Table 10)

\section{DISCUSSION}

The study was conducted to perceive and interpret the current health status and disease burden of children in remote rural pockets of Himachal Pradesh in and around districts of Solan. We also wanted to assess indirectly the impact of different health programs, how far we have reached since these programs are in place under different umbrellas since long, like iron \& folic acid supplementation, periodic deworming, mid-day meal etc., which are currently under national health mission, to improve the health status of children.[14] We found that anaemia is still widely prevalent in children and as it effects their cognitive development, growth and immunity, it is of major concern. Anaemia effects the productivity of nation in long term and scholastic performance of children therefore it needs to be addressed more meticulously individually and as a society also. The recent launch of Potion Abhiyaan[15]and Anaemia mukt Bharat[16] for 2018-2020 has set specific goals to be achieved in particular and are also working to achieve the set goals in national health policy by 2025 but the problem is found to be more in our study as compared to national figures varying from $30-69 \%$ as per NFHS- 4 data $2015 / 16 .[17,18]$

Protein energy malnutrition is also prevalent with about $25 \%$ children in severe grade, but there was no case of kwashiorkor or PEM with oedema. Presentation was subacute in nature with clinical and subclinical effects on growth and development, recognized mainly by anthropometric measurements. We recommend that actionoriented measurements should be taken in this regard. The recent global trend of overweight and obesity was largely absent and appears to be not a public problem in rural hills.[19] No case of alcohol, tobacco or drug abuse was found in adolescents. [20]

Since this population lived in a difficult hilly terrain, we specially looked for children who had uncommon or rare findings, congenital or genetic diseases who need long term special care, family support, and who might be an economic burden to family. Surprisingly we found a larger than expected number of children hidden in these rural pockets deprived of special care, genetic testing or counselling etc.[21]

A shift in approach in remote areas from communicable diseases to noncommunicable diseases is the need of hour as the relative lack of communication and commutation facilities apparently has helped in prevention of spread of communicable diseases in these areas but they are not without problems rather marked with problems of different nature.

A look out approach for congenital/ Genetic/ uncommon/ rare diseases was kept, including involvement of central nervous system to direct future policies and development of treatment modalities in a sector which remains secluded, unaddressed and is not only missing in national policies but also at global level think tank scenario.

Interest has developed recently[22] to address these issues globally. An alarmingly high number of such hidden cases were detected comprising a total number of 38 which was $8.05 \%$. Clinical diagnosis included Down Syndrome, Sturge weber Syndrome, Cerebral palsy, Global developmental delay, cavernous haemangioma, learning disabilities, Ventricular septal defect, Growth hormone deficiency and hypothyroid state etc., which need long term regular specialist care and sophisticated investigations or Chromosomal analysis.

Rural areas are marked by delay in seeking proper healthcare due to poverty, ignorance and challenging geographical terrain, non-availability of easy and affordable transport, with high economic burden both in terms of expenditure and loss of wages.[23] The type of disease also determines the health seeking behaviour, diseases being chronic in nature, subacute in presentation sometimes marked with poor prognosis and poor outcome, also make acceptance of problem as God given but they cannot be left unattended.

The most frequent complaint was Pain and among pain abdominal pain $(n=62)$ topped the list. (Table No. 6) Other gastrointestinal problems were also significant in number. Other studies have also found pain abdomen as the commonest complaint in children. Respiratory problems and skin problems closely followed. Fever was down the list with $\mathrm{n}=37$ cases. It is to be noted that there is considerable unmet disease burden in these rural pockets of hills. Patients usually had multiple complaints and chronicity, growth problems were brought as single complaints in few only $(n=23)$, which means the problem largely remained unnoticed and unrecognized and unattended by parents.

Highest burden of disease was in category ICD chapter 3, diseases of blood and blood forming organs and certain disorders involving the immune system due to high anaemia burden, which has been recognized nationally and by WHO also, but our study indicates it to be further higher. Next was chapter 4 i.e., endocrine, behavioural and metabolic diseases. These problems areas are so well recognized in children in hospital-based studies although not well addressed in campbased studies. We have brought them in focus for current action and future reference. Problem of Malnutrition, Growth and development issues in children are of major concern for any society as they interfere in achievement of true potential of children both physically and mentally. Children are the future of any nation and hence prevalence of significant number of these problems as found in our study is an eye opener as these problems are still prevalent in abundance in 
rural pockets of Himachal and need to be addressed more vigorously.

\section{CONCLUSIONS}

The study has addressed child health comprehensively and will help as baseline in comparing and formulating the policies in future. There is a considerable hidden disease burden in rural pockets of Himachal Pradesh with a subacute or chronic presentation. There is substantial delay in health seeking behaviour of rural population and specialist care is almost totally missing even in days of super specialty approach globally. Early identification and early intervention approach to improve the situation and prevent permanent disabilities and progression of disease needs to be formulated to influence the health seeking behaviour of rural parents and children as well. A major shift from clinic and hospital-based service to an outreach approach in their own rural environment is needed to affect their health seeking behaviour. As nutrition plays a big role in child health and affects their growth and development, more effort and directives are needed to help children achieve their full potential not only through supplementation policies but also through investigative approach for correction. A paradigm shift in approach to treat noncommunicable diseases especially in areas where communicable diseases are relatively absent is recommended with formulation of area specific policies. Uncommon and rare diseases including CNS diseases are hidden neglected and unaddressed. We have to direct our efforts to address this challenging issue not only at research levels but also at improving awareness \& management at community level.

\section{REFERENCES}

[1] Ashoka Change makers. URL: http://www.steps.org.in/. Accessed November 10, 2016.

[2] Americares India. URL: http://www.americaresindia.org/whatwedo/mobilemedical-camps. Accessed November 10, 2016.

[3] Smile foundation India. URL: http://www.smilefoundationindia. Accessed November 10, 2016.

[4] Common childhood concerns in Indian Children. URL: http://www.indiaparenting.com/childs-healthcare. Accessed November 10, 2016.

[5] District level Household and facility Survey - 4, state fact sheet Himachal Pradesh (2012-13), International Institute for Population sciences. URL: http://www.iipsindia.org Accessed November 10, 2016.

[6] National Health Programmes. URL http://www.mohfw.nic.in/index1.Accessed November $12,2016$.
[7] CNS diseases world summit 2016 overview. URL https://www.gtcbio.com/conferences/cns-diseasesworld-summit-overview. Accessed November 12, 2016.

[8] NCMH background papers: Burden of diseases in India URL: http://www.who.int/macrohealth/action/NCMH

[9] Mohamed IN, Elseed MA, Hamed AA. Clinical profile of pediatric neurological disorders: outpatient department, Khartoum, Sudan. Child Neurol Open 2016;3: 2329048X15623548.

[10] Merikangas KR, Nakamura EF, Kessier RC. Epidemiology of mental disorders in children and adolescents. Dialogues Clin Neurosci 2009;11(1):7-20.

[11] Khadilkar SV. Neurology: the scenario in India. J Assoc Physicians India 2012;60:42-4.

[12] Navaneetham D. Rare diseases and disordersestimated rare diseases population in South Asian Countries. https://www.rarediseaseindia.org Accessed November 12, 2016.

[13] Haemoglobin concentrations for the diagnosis of anaemia and assessment of severity. http://www.who.int/vmnis/indicators/haemoglobin. pdf?ua $=1 \mathrm{WHO} / \mathrm{NMH} / \mathrm{NHD} / \mathrm{MNM} / 11.1 . \quad$ Accessed November 12, 2016.

[14] Kotecha PV. Nutritional anaemia in young children with focus on Asia and India. Indian J Community Med 2011;36(1):8-16.

[15] National family health survey 2015-16 report. http://rchiips.org/nfhs/factsheet_nfhs-4.shtml Accessed April 142019.

[16] Nutrition factsheet, Poshan Abhiyan. India and Anaemia Mukt Bharat https://www.nutritionindia.info/ Accessed April 14 2019.

[17] Overview of anaemia prevalence. https://anaemiamuktbharat.info/dashboard/\#/ Accessed April 142019.

[18] Grantham-McGregor S, Ani C. A review of studies on the effect of Iron deficiency on cognitive development in children. J Nutr 2001;131(2S-2):649S-68S. http://jn.nutrition.org/content/131/2/649S.

[19] Sahoo K, Sahoo B, Choudhary AK, et al. Childhood obesity: causes and consequences: J Family Med Prim Care 2015;4(2):187-92.

[20] Rahman F, Tripathi VN. Substance abuse among male adolescents in northern India: International Journal of Contemporary Pediatrics 2016;3(2):495-7.

[21] Rare diseases: Facts and Statistics. https://www.globalgenes.org Accessed November 12, 2016.

[22] Reardon W, Donnai D. Dysmorphology demystified. Arch Dis Child Fetal Neonatal Ed 2007;92(3):F225-9.

[23] Pambos M, Jessica Ng, Loukes J, et al. Demographics and diagnoses at rural health camps in Nepal: crosssectional study. Fam Pract 2012;29(5):528-33. 